Mon. Not. R. Astron. Soc. 000, 112 (2012) Printed 18 July $2018 \quad$ (MN LATEX style file v2.2)

\title{
Instability of Superfluid Flow in the Neutron Star Core
}

\author{
B. Link ${ }^{\star}$ \\ Department of Physics, Montana State University, Bozeman, MT 59717, USA
}

18 July 2018

\begin{abstract}
Pinning of superfluid vortices to magnetic flux tubes in the outer core of a neutron star supports a velocity difference of $\sim 10^{5} \mathrm{~cm} \mathrm{~s}^{-1}$ between the neutron superfluid and the proton-electron fluid as the star spins down. Under the Magnus force that arises on the vortex array, vortices undergo vortex creep through thermal activation or quantum tunneling. We examine the hydrodynamic stability of this situation. Vortex creep introduces two low-frequency modes, one of which is unstable above a critical wavenumber for any non-zero flow velocity of the neutron superfluid with respect to the charged fluid. For typical pinning parameters of the outer core, the superfluid flow is unstable over wavelengths $\lambda \lesssim 10 \mathrm{~m}$ and over timescales of $\sim(\lambda / 1 \mathrm{~m})^{1 / 2}$ yr down to $\sim 1 \mathrm{~d}$. The vortex lattice could degenerate into a tangle, and the superfluid flow would become turbulent. We suggest that superfluid turbulence could be responsible for the red timing noise seen in many neutron stars, and find a predicted spectrum that is generally consistent with observations.
\end{abstract}

Key words: hydrodynamics - turbulence - stars: neutron - pulsars: general - stars: rotation.

\section{INTRODUCTION}

The dynamical role of hydrodynamic instabilities in the liquid core of a neutron star is of considerable interest in understanding a variety of phenomena that includes spin glitches, stochastic spin variations ("timing noise") and thermal evolution, as well as possible precession and r-modes. One instability that could arise is the "Glaberson-Donnelly" counterflow instability observed in superfluid liquid helium, wherein flow of the fluid's normal component along the vortices that thread a rotating superfluid drives the system turbulent (Glaberson et al. 1974). Early in a neutron star's evolution, when the temperature of the core is comparable to the condensation temperature of the neutrons, differential rotation in the core, resulting from a glitch or possibly causing it, drives an Ekman flow along the rotation axis that can excite the Glaberson-Donnelly instability (Peralta et al. 2005, 2006). Peralta et al. (2006) and Melatos \& Peralta (2007) have shown that transitions between laminar flow and fully-developed turbulence could drive spin glitches. Unstable shear layers in the outer core may also play a role in glitches (Peralta \& Melatos 2009).

The protons of the outer core are predicted to form a type II superconductor (Migdal 1959). When the protons condensed early in the star's life, the expulsion time for magnetic flux was far greater than the nucleation time of magnetic flux tubes, and the magnetic flux became confined to flux tubes (Bavm et al. 1969). The magnetic field inside a flux tube is comparable to the lower critical field for superconductivity, $B_{c} \sim 10^{15} \mathrm{G}$. Entrainment of the proton and neutron mass currents causes magnetization of the neutron vortices with a field in the vortex core comparable to $B_{c}$ (Alpar et al. 1984b). If the vortices are free to move, momentum exchange between the charged fluid and the neutron superfluid through the scattering of electrons with the magnetized vortex cores quickly damps velocity differences between the two fluids. The strong magnetization of the vortex cores, however, will pin the vortices to the flux tubes with a pinning energy of $\gtrsim 100 \mathrm{MeV}$ per intersection (see Jones 1991; Mendell 1991; Chau et al. 1992; Ruderman et al. 1998, and the estimates of $\S 2$ below). Pinning plays a crucial role in the dynamics of the core fluids; to the extent that pinning is perfect, the motions of the two fluids are largely independent, apart from modifications to the average mass currents by the entrainment effect.

\footnotetext{
^ E-mail: link@physics.montana.edu;
} 
Sidery et al. (2008) and Glampedakis et al. (2008b a) have studied a variant of the Glaberson-Donnelly instability for the core mixture of superfluid neutrons, superconducting protons, and electrons. The proton-electron liquid acts analogously to the normal component in liquid helium. They found that inertial waves of the neutron superfluid are subject to the Glaberson-Donnelly instability if there is a relative flow between the proton-electron fluid and the neutron fluid directed along the rotation axis, whether pinning is negligible (Siderv et al. 2008) or perfect (Glampedakis et al. 2008b. a). Such a relative flow could result in a precessing neutron star in which pinning of vortices to flux tubes is effective. van Hoven \& Levin (2008) have shown, however, that the enormous magnetic stress in the charged fluid suppresses the instability for relative flow speeds less than the hydromagnetic wave speed; the instability is unlikely to occur, even in a precessing neutron star.

Andersson et al. (2004) have shown that relative motion between the neutron fluid and the charged fluid can be susceptible to a two-stream instability, though pinning was not considered. This instability is similar to the Kelvin-Helmholtz instability for relative flow of two fluids separated by an interface, but occurs when the fluids coexist in the same volume. Glampedakis \& Andersson (2009) have identified a instability that can occur in the neutron-proton mixture of the core associated with inertial r-modes, assuming perfect pinning of vortices to flux tubes. The instability occurs if the relative angular velocity difference between the proton and neutron liquids everywhere exceeds a critical value. Glampedakis \& Andersson (2009) propose that this instability triggers glitches, though it has not been explained how the the critical angular velocity difference for instability reaches its critical value nearly everywhere in the star at once, or how a glitch is subsequently triggered.

From the standpoint of building realistic rotational models of neutron stars, a crucial question is if laminar flow in the star is generally stable or unstable. As a first step towards answering this question, we studied the stability of the vortex lattice, pinned to the nuclei of the inner crust, in the presence of the superfluid flow sustained by pinning as the star spins down (Link 2012). For the situation of imperfect pinning that would arise as a result of, for example, vortex motion through thermal activation or quantum tunneling, two low-frequency modes appear in the system, one of which is unstable over length scales of less than $\sim 10 \mathrm{~m}$, and over timescales possibly as fast as $\sim 100 \mathrm{~d}$. We suggested that the instability could lead to turbulence similar to grid turbulence seen in liquid helium (Smith et al. 1993). In this paper, we extend the analysis to the neutron star core and find an instability that is analogous to that found in crust. As the star spins down, the Magnus force on the pinned vortices exerts stresses on the neutron and charged fluids that drives the flow unstable, possibly over timescales as short as days.

In $\S 2$, we estimate the angular velocity difference between the neutron fluid and the charged fluid that can be sustained by pinning in a spinning-down neutron star. In $\S 3$, we examine the stability of this state of differential rotation, and show that it is unstable. In $\S 4$, we estimate the spectrum of stochastic torque that would arise if the fluid becomes turbulent, and compare to measured power spectra in radio pulsars and magnetars. Our chief conclusion is that the superfluid in a spinning-down neutron star is generally turbulent everywhere that there is significant pinning, hence, throughout the outer core and inner crust.

\section{PINNING OF VORTICES TO FLUX TUBES}

We begin by establishing the approximate density range of type II superconductivity, where the magnetic field will be confined to flux tubes and the pinning of vortices to flux tubes will be important. The proton fluid will be a type II superconductor when the proton coherence length $\xi_{p}$ and the London length $\Lambda_{*}$ satisfy

$\xi_{p}<\sqrt{2} \Lambda_{*}$

The London length is (Alpar et al. 1984b)

$\Lambda_{*}=30\left[\frac{m_{p}^{*}}{m_{p}} x_{p}^{-1} \rho_{14}^{-1}\right]^{1 / 2} \mathrm{fm}$,

where $m_{p}$ is the bare neutron mass and $m_{p}^{*}$ is its effective mass in the medium, $x_{p} \equiv \rho_{p} / \rho_{n}, \rho_{p}$ and $\rho_{n}$ are the mass densities of protons and neutrons, and $\rho_{14}$ is the total mass density in units of $10^{14} \mathrm{~g} \mathrm{~cm}^{-3}$. In the outer core, $m_{p}^{*} / m_{p} \simeq 1 / 2($ Siöberg 1976; Chamel \& Haensel 2006).

The proton coherence length $\xi_{p}$ is (Mendell 1991)

$\xi_{p}=16 x_{p}^{1 / 3} \rho_{14}^{1 / 3} \frac{m_{p}}{m_{p}^{*}} \Delta_{p}(\mathrm{MeV})^{-1} \mathrm{fm}$,

where $\Delta_{p}$ is the proton pairing gap. The core begins at a density of $\rho_{14} \simeq 1.5$ (Ravenhall et al. 1983 ), and the proton gap $\Delta_{p}$ has a typical value of $\sim 1 \mathrm{MeV}$ around nuclear density $\rho_{14}=2.8$ (Elgarøy et al. 1996). Combining eqs. (2) and (3) gives

$\frac{\xi_{p}}{\sqrt{2} \Lambda_{*}} \simeq 0.3\left(\frac{m_{p}^{*} / m_{p}}{0.5}\right)^{-3 / 2}\left(\frac{x_{p}}{0.05}\right)^{5 / 6}\left(\frac{\rho_{14}}{4}\right)^{5 / 6} \Delta_{p}(\mathrm{MeV})^{-1}$ 
The protons thus form a type II superconductor in the outer core. Above nuclear density the proton gap begins to fall (Elgarøy et al. 1996), and a transition to a type I superconductor occurs at several times nuclear density. Polarization effects may lower $\Delta_{p}$ by a factor of 2-3 (Schulze et al. 1996), restricting the type II region to somewhat lower density.

Vortices and flux tubes interact with one another and pin as a result of the interaction between the neutron and proton condensates. For neutrons flowing at velocity $\boldsymbol{v}_{n}$ and protons at velocity $\boldsymbol{v}_{p}$, the mass currents take the form (Alpar et al. 1984b)

$\boldsymbol{g}_{p}=\rho_{p p} \boldsymbol{v}_{p}+\rho_{p n} \boldsymbol{v}_{n}, \quad \boldsymbol{g}_{n}=\rho_{n n} \boldsymbol{v}_{p}+\rho_{n p} \boldsymbol{v}_{n}$,

The mass current of each species is generally in a direction different than the velocity of either species. This entrainment effect is fundamentally non-dissipative.

For a proton mass density $\rho_{p}$ and neutron mass density $\rho_{n}$, the coefficients for the mass currents are given by

$\rho_{p p}=\rho_{p}\left(\frac{m_{p}}{m_{p}^{*}}\right) \quad \rho_{n n}=\rho_{n}\left(\frac{m_{n}}{m_{n}^{*}}\right) \quad \rho_{n p}=\rho_{p n}=\rho_{p}\left(\frac{\delta m_{p}^{*}}{m_{p}}\right)=\rho_{n}\left(\frac{\delta m_{n}^{*}}{m_{n}}\right)$,

where $m_{n}^{*}$ is the effective mass of the neutron; $\delta m_{p}^{*} \equiv m_{p}^{*}-m_{p}$ and $\delta m_{n}^{*} \equiv m_{n}^{*}-m_{n}$ are the contributions to the effective masses due to interactions in the medium. The neutron and proton mass densities, and the total density, neglecting the electron mass, are

$\rho_{p}=\rho_{p p}+\rho_{p n} \quad \rho_{n}=\rho_{n n}+\rho_{n p} \quad \rho=\rho_{n}+\rho_{p}$.

In the outer core, $\delta m_{p}^{*} / m_{p} \simeq-1 / 2$ and $m_{n}^{*} / m_{n} \simeq 1$ (Siöberg 1976; Chamel \& Haensel 2006), and $\rho_{p n}$ is negative.

Ruderman et al. (1998), using Ginzburg-Landau theory and the results of Alpar et al. (1984b), have calculated the energy per unit length $L$ of a superimposed flux tube and vortex line, minus the energy for infinite separation:

$\frac{E_{0}}{L} \simeq \frac{\pi}{8}\left(\frac{\Phi_{0}}{\pi \Lambda_{*}^{2}}\right)^{2} \Lambda_{*}^{2} \frac{m_{p}}{m_{n}} \frac{\rho_{p n}}{\rho_{p p}} \ln \left(\frac{\Lambda_{*}}{\xi}\right)=\frac{\pi}{8} B_{v} B_{\Phi} \Lambda_{*}^{2} \ln \left(\frac{\Lambda_{*}}{\xi_{n}}\right)$

where $\Phi_{0} \equiv h c / 2 e$ is the flux quantum, $B_{\Phi} \equiv \Phi_{0} / \pi \Lambda_{*}^{2}$ is the characteristic magnetic field in a flux tube core, $B_{v} \equiv$ $\left(\Phi_{0} / \pi \Lambda_{*}^{2}\right)\left(m_{p} \rho_{p n} / m_{n} \rho_{p p}\right)$ is the field in the vortex core, and $\xi_{n}$ is the neutron coherence length. Since $\rho_{p n}$ is negative, $E / L$ is negative if the vorticity is parallel to $\boldsymbol{B}_{v}$, and positive if anti-parallel. The interaction is primarily magnetic. Both the flux tube and the vortex line have their magnetic fields screened over the London length $\Lambda_{*}$.

If the angle between the vortex line and the flux tube is $\theta$, the overlap length is $l \simeq 2 \Lambda_{*} / \sin \theta$. The interaction energy per vortex-flux tube junction is approximately

$E_{p}(\theta) \simeq l \frac{E_{0}}{L}=\frac{\pi}{8} \boldsymbol{B}_{v} \cdot \boldsymbol{B}_{\Phi}\left(\Lambda_{*}^{2} l\right) \ln \left(\frac{\Lambda_{*}}{\xi_{n}}\right)=\frac{\pi}{4}\left(\frac{\Phi_{0}}{\pi \Lambda_{*}^{2}}\right)^{2} \Lambda_{*}^{3} \frac{\delta m_{p}^{*}}{m_{p}} \ln \left(\frac{\Lambda_{*}}{\xi_{n}}\right) \cot \theta$

This equation provides only a rough estimate for arbitrary $\theta$, since eq. (8) does not account for modifications of vortex and flux tube structure for $\theta \neq 0$. The pinning energy is approximately the magnetic energy density in the overlap region, times the overlap volume $\Lambda_{*}^{2} l$. Similar estimates have been obtained by Jones (1991), Mendell (1991), and Chau et al. (1992).

The magnetic field is expected to be highly tangled (Ruderman et al. 1998), and so $\theta$ could take a broad range of values. For an average radius of curvature of flux tubes of $R_{\Phi}$, the maximum overlap length is

$l_{\max }=2 R_{\Phi} \cos ^{-1}\left(1-\Lambda_{*} / R_{\Phi}\right) \equiv \frac{2 \Lambda_{*}}{\sin \theta_{\min }}$,

where $\theta_{\min }$ is the minimum angle at which a curved flux tube intersects a straight vortex. For $R_{\Phi}>>\Lambda_{*}$, this equation gives $\theta_{\min } \simeq\left(2 \Lambda_{*} / R_{\Phi}\right)^{1 / 2}$. The interaction is repulsive for $\pi / 2<\theta<\pi$, but the magnitude of the pinning interaction is the same for both $\theta$ and $\pi-\theta$. Vortices will be immobilized by flux tubes in much the same way regardless of the sign of the interaction. We average $\left|E_{p}(\theta)\right|$ over $\theta$ to obtain the average pinning energy for $R_{\Phi}>>\Lambda_{*}$

$\left\langle E_{p}\right\rangle=\frac{1}{\left(\pi-2 \theta_{\min }\right)} \int_{\theta_{\min }}^{\pi-\theta_{\min }} d \theta\left|E_{p}(\theta)\right| \simeq \frac{2}{\pi} E_{p}(\cot \theta=1) \ln \sqrt{\frac{R_{\Phi}}{2 \Lambda_{*}}}$.

Unless $R_{\Phi}$ is much larger than $\sim 10^{3} \Lambda_{*}$, the logarithmic factor is not significantly different than one, so we henceforth set this factor to unity. If $R_{\Phi}$ is much larger, however, it is probably a better approximation to set $\theta$ equal to the inclination angle between the star's magnetic moment and the rotation axis. In either case eq. (11), with the logarithmic factor set to unity, should be a good estimate.

The pinning energy per vortex-flux tube junction is, taking a typical value $\ln \left(\Lambda_{*} / \xi_{n}\right)=0.5$,

$\left\langle E_{p}\right\rangle \simeq 10^{2}\left(\frac{m_{p}^{*} / m_{p}}{0.5}\right)^{-1 / 2}\left(\frac{\left|\delta m_{p}^{*}\right| / m_{p}}{0.5}\right)\left(\frac{x_{p}}{0.05}\right)^{1 / 2}\left(\frac{\rho_{14}}{4}\right)^{1 / 2} \mathrm{MeV}$, 
The pinning force is $F_{p} \sim\left\langle E_{p}\right\rangle / \Lambda_{*}$, typically $\sim 1 \mathrm{MeV} \mathrm{fm}^{-1}$.

For a single vortex immersed in a tangle of flux tubes, the average length between intersections will equal the average distance between flux tubes, $l_{\Phi}=n_{\Phi}^{-1 / 2}$, where $n_{\Phi}=B / \Phi_{0}$ is the areal density of flux tubes and $B$ is the average magnetic field strength. The pinning force per unit length is $f_{p}=F_{p} / l_{\Phi}$. For a velocity difference $w$ between the neutron superfluid and the pinned vortex, the fluid flow exerts a Magnus force per unit length $\rho_{n} \kappa w$, where $\kappa \equiv h / 2 m_{n}$ is the vorticity quantum. The critical velocity difference $w_{c}$ that can be sustained is given by

$f_{p}=\frac{\left\langle E_{p}\right\rangle}{\Lambda_{*} l_{\Phi}}=\rho_{n} \kappa w_{c}$

so that

$w_{c} \sim 10^{5}\left(\frac{x_{p}}{0.05}\right)\left(\frac{m_{p}^{*} / m_{p}}{0.5}\right)^{-1}\left(\frac{\left|\delta m_{p}^{*}\right| / m_{p}}{0.5}\right) B_{12}^{1 / 2} \mathrm{~cm} \mathrm{~s}^{-1}$,

where $B_{12}$ is the strength of the field in units of $10^{12} \mathrm{G}$. The critical velocity is not a strong function of density.

As a star spins down, pinning prevents the neutron superfluid from corotating with the charged fluid, and a velocity difference $w$ builds. If $w \simeq 0$ at some time, spin down of the crust will cause the critical velocity to be reached in a time $\Delta t$ that is short compared to the star's age:

$\Delta t \simeq \frac{w_{c}}{R 2 \pi|\dot{\nu}|}=30\left(\frac{w_{c}}{10^{5} \mathrm{~cm} \mathrm{~s}^{-1}}\right)\left(\frac{t_{\text {age }}}{10^{4} \mathrm{yr}}\right)\left(\frac{\nu}{10 \mathrm{~Hz}}\right)^{-1} \mathrm{yr}$,

where $\nu$ is the spin frequency of the crust, $t_{\text {age }} \equiv \nu / 2 \dot{\nu}$ is the spin-down age, and $R$ is the stellar radius. Hence, the angular velocity difference between the superfluid and the charged components will be $w_{c} / R \sim 10^{-1} \operatorname{rad~s}^{-1}$ throughout the star's life (except, for example, right after a glitch). We now examine the stability of this state of differential rotation.

\section{STABILITY ANALYSIS}

The problem of the coupled dynamics of the neutron and proton fluids can be treated with hydrodynamics for length scales that are large compared to the inter-vortex spacing $l_{v}$, restricting the treatment to wavenumbers $k l_{v}<<1$. In a single-component superfluid, the vortex lattice supports Tkachenko modes of speed $c_{T}=(\hbar \Omega / 4 m)^{1 / 2}$ (Tkachenko $1966 \mathrm{a}$. b) , where $\Omega$ is the spin rate of the superfluid and $m$ is the mass of the fundamental boson, twice the neutron mass for a neutron superfluid. (For studies of Tkachenko modes in neutron stars and the effects of dissipation on these modes, see Noronha \& Sedrakian 2008 and Haskell 2011). For a typical neutron star, $c_{T} \simeq 10^{-1}\left(\Omega / 100 \mathrm{rad} \mathrm{s}^{-1}\right)^{1 / 2} \mathrm{~cm} \mathrm{~s}^{-1}$. The degrees of freedom of the vortex lattice can be ignored to a very good approximation when the speed of the background flow with respect to pinned vortices is much larger than $c_{T}(\operatorname{Link} 2012)$, as is the case for vortex pinning to flux tubes. The areal density of vortices is $l_{v}^{-2}=2 m \Omega / h$ for a uniform vortex lattice; hence, the requirement that $k l_{v}<<1$ is equivalent to $c_{T} k<<\Omega$.

In the laboratory frame, the acceleration equations for neutrons flowing at velocity $\boldsymbol{v}_{n}$ and protons plus electrons flowing at velocity $\boldsymbol{v}_{p}$, are (Prix 2004; Andersson \& Comer 2006; Glampedakis et al. 2008b; van Hoven \& Levin 2008; Glampedakis et al. 2011)

$\left(\partial_{t}+\boldsymbol{v}_{n} \cdot \nabla\right)\left(\boldsymbol{v}_{n}-\epsilon_{n} \boldsymbol{w}_{n p}\right)-\epsilon_{n} w_{n p}^{i} \nabla v_{i}^{n}=-\nabla \mu_{n}+\boldsymbol{f} / \rho_{n}$

$\left(\partial_{t}+\boldsymbol{v}_{p} \cdot \nabla\right)\left(\boldsymbol{v}_{p}+\epsilon_{p} \boldsymbol{w}_{n p}\right)+\epsilon_{p} w_{n p}^{i} \nabla v_{i}^{p}=-\nabla \mu_{p}-\boldsymbol{f} / \rho_{p}+\nu_{e} \nabla^{2} \boldsymbol{v}_{p}+\left(1 / 4 \pi \rho_{p}\right) \boldsymbol{B}_{\mathrm{eff}} \cdot \nabla \boldsymbol{B}_{\mathrm{eff}}$,

where $\boldsymbol{w}_{n p} \equiv \boldsymbol{v}_{n}-\boldsymbol{v}_{p}, i$ is a coordinate index and is summed, $\mu_{n}$ and $\mu_{p}$ are the neutron and proton chemical potentials, $\rho_{n}$ and $\rho_{p}$ are the neutron and proton mass densities (neglecting the electron mass), $\boldsymbol{f} / \rho_{n}$ is the force per unit volume exerted on the neutron fluid by the proton-electron fluid, $\nu_{e}$ is the electron kinematic shear viscosity, and $B_{\text {eff }} \equiv \sqrt{B B_{c}}$, where $B$ is the average field strength and $B_{c} \simeq 10^{15} \mathrm{G}$ is the value of the lower critical field. Electron-electron scattering gives the dominant contribution to the viscosity, as all other scattering processes are strongly suppressed when both the neutrons and protons are superfluid. The terms proportional to $\epsilon_{n}$ and $\epsilon_{p}$ account for entrainment between neutrons and protons, and are related by $\rho_{n} \epsilon_{n}=\rho_{p} \epsilon_{p}$. We take the entrainment coefficients to be constants. The small effects of electron inertia and the London current, both neglected here, have been considered by Glampedakis et al. (2011).

For the highly-conductive proton-electron fluid, the magnetic field is frozen to the proton-electron fluid. The field obeys the induction equation

$\partial_{t} \boldsymbol{B}+\nabla \times\left(\boldsymbol{B} \times \boldsymbol{v}_{p}\right)=0$.

The force per unit volume on the neutron fluid, $\boldsymbol{f} / \rho_{n}$, is equal to the Magnus force per unit volume on the vortex array, that is,

$\boldsymbol{f} / \rho_{n}=\boldsymbol{\omega}_{n} \times\left(\boldsymbol{v}_{n}-\boldsymbol{v}_{v}\right)$ 
where $\boldsymbol{v}_{v}$ is the local vortex velocity and $\boldsymbol{\omega}_{n} \equiv \nabla \times \boldsymbol{v}_{n}$ is the local vorticity of the neutron superfluid. For perfect pinning of vortices against flux tubes, $\boldsymbol{v}_{v}=\boldsymbol{v}_{p}$. The vortex velocity with respect to the proton-electron fluid has a component along $\boldsymbol{w}_{n p} \equiv \boldsymbol{v}_{n}-\boldsymbol{v}_{p}$, and a component orthogonal to both $\boldsymbol{w}_{n p}$ and $\boldsymbol{\omega}_{n}$. The local vortex velocity can thus be written as

$\boldsymbol{v}_{v}=\boldsymbol{v}_{p}+\left(1-\beta^{\prime}\right) \boldsymbol{w}_{n p}+\beta \boldsymbol{w}_{n p} \times \hat{\omega}_{n}$,

where $\beta^{\prime}$ and $\beta$ are coefficients that determine the vortex mobility, and we assume to be constant. Perfect pinning of vortices to the charged fluid corresponds to $\beta^{\prime}=1$ and $\beta=0$. The force, from eq. (19), is

$\boldsymbol{f} / \rho_{n}=\beta^{\prime} \boldsymbol{\omega}_{n} \times \boldsymbol{w}_{n p}+\beta \hat{\omega}_{n} \times\left(\boldsymbol{\omega}_{n} \times \boldsymbol{w}_{n p}\right)$.

This force is the mutual friction force introduced in by Hall \& Vinen (1956), but appearing here in a different context. Imperfect pinning, that is, "vortex creep", corresponds to $\alpha \equiv 1-\beta^{\prime}<<1$ and $\beta<<1$. We refer to $\alpha$ and $\beta$ as the "pinning coefficients". Perfect pinning corresponds to the limit $\alpha=\beta=0$, while no pinning $(\boldsymbol{f}=0)$ corresponds to $\alpha=1$ and $\beta=0$. Vortices move with a component along $\boldsymbol{w}_{n p}$, so that $0<\alpha \leq 1$. The energy dissipation rate per unit volume is determined by $\beta$, which must be positive to give local entropy production. More general dissipative forces have been considered by Andersson \& Comer (2006).

Vortex creep could be a low-drag process, with $\beta<<\alpha$, or a high-drag process, with $\beta>>\alpha$. In much previous work on pinning, the high-drag limit has been implicitly assumed through the following relationship between $\beta$ and $\beta^{\prime}$ :

$\beta^{\prime}=1-\alpha=\frac{\mathcal{R}^{2}}{1+\mathcal{R}^{2}}=\mathcal{R} \beta$,

where $\mathcal{R}$ is a dimensionless drag coefficient. In this drag description, imperfect pinning corresponds to $\mathcal{R}>>1$ so that eq. (22) requires $\beta>>$. Eq. (22) is not true in general, since the presence of non-dissipative forces between vortices and the charged fluid can give $\beta<<\alpha$, a regime of low drag (Link 2009). As we show below, it is the low-drag regime that is likely to be realized, with vortex creep being unstable in this regime. A crucial feature of our analysis is that we do not impose eq. (22) for imperfectly-pinned vortices. We will assume in $\S 4$ that eq. (22) holds only for the tiny fraction of vortex length $\left(\sim 10^{-6}\right)$ that is unpinned at any instant.

To examine the stability of the flow with imperfect pinning, we use a local plane wave analysis in the frame rotating with the unperturbed charged fluid at angular velocity $\boldsymbol{\Omega}_{0}$, in which $\boldsymbol{v}_{p}=0$ and the unperturbed flow velocity arising from spin down of the charged fluid is $\boldsymbol{w}_{0}=\boldsymbol{w}_{n p, 0}=w_{0} \hat{x}$. Restricting the analysis to the regime $k R \gtrsim 1$, where $R$ is the stellar radius, the background flow can be treated as approximately uniform. Let the rotation axis $\hat{z}, \boldsymbol{k}$, and $\boldsymbol{w}_{0}$ all be coplanar, with an angle $\theta$ between $\boldsymbol{k}$ and the rotation axis. The angle between $\hat{z}$ and $\boldsymbol{B}$ is $\theta_{B}$. We restrict the analysis to the quadrant $0 \leq \theta \leq \pi / 2$. The linearized equations of motion in the rotating frame are:

$\left(\partial_{t}+\boldsymbol{w}_{0} \cdot \nabla\right)\left(\delta \boldsymbol{v}_{n}-\epsilon_{n} \delta \boldsymbol{w}_{n p}\right)-\epsilon_{n} w_{0} \nabla\left(\hat{x} \cdot \delta \boldsymbol{v}_{n}\right)-2 \boldsymbol{\Omega}_{0} \times \delta \boldsymbol{v}_{n}=-\nabla \delta \mu^{\prime}+\delta \boldsymbol{f} / \rho_{n}$

$\nabla \cdot \delta \boldsymbol{v}_{n}=0$

$\partial_{t}\left(\boldsymbol{v}_{p}+\epsilon_{p} \delta \boldsymbol{w}_{n p}\right)+\epsilon_{p} w_{0} \nabla\left(\hat{x} \cdot \delta \boldsymbol{v}_{n}\right)+2 \boldsymbol{\Omega}_{0} \times \boldsymbol{v}_{p}=-\nabla \delta \mu_{p}^{\prime}-\delta \boldsymbol{f} / \rho_{p}+\nu_{e} \nabla^{2} \boldsymbol{v}_{p}+\frac{\boldsymbol{B}_{\mathrm{eff}, 0}}{4 \pi \rho_{p}} \cdot \nabla \delta \boldsymbol{B}_{\mathrm{eff}}$

$\delta \boldsymbol{f} / \rho_{n}=(1-\alpha) \delta\left\{\boldsymbol{\omega}_{n} \times \boldsymbol{w}_{n p}\right\}+\beta \delta\left\{\hat{\omega}_{n} \times\left(\boldsymbol{\omega}_{n} \times \boldsymbol{w}_{n p}\right)\right\}$.

$\nabla \cdot \boldsymbol{v}_{p}=0$

$\partial_{t} \delta \boldsymbol{B}+\nabla \times\left(\boldsymbol{B}_{0} \times \boldsymbol{v}_{p}\right)=0$

where $\delta$ denotes a perturbed quantity, and $\delta \boldsymbol{v}_{p}=\boldsymbol{v}_{p}$. Here $\mu_{n, p}^{\prime} \equiv \mu_{n, p}-\rho_{n, p}\left(\boldsymbol{\Omega}_{0} \times \boldsymbol{r}\right)^{2} / 2$. The vorticity appearing in this equation is the total vorticity evaluated in the laboratory frame, and is only slightly larger than $2 \Omega_{0}$ :

$\frac{\omega_{0}-2 \Omega_{0}}{2 \Omega_{0}} \simeq \frac{w_{0}}{R \Omega_{0}}=10^{-3}\left(\frac{w_{0}}{10^{5} \mathrm{~cm} \mathrm{~s}^{-1}}\right)\left(\frac{\Omega_{0}}{100 \mathrm{rad} \mathrm{s}^{-1}}\right)^{-1}$.

We henceforth take $\omega_{0}=2 \Omega_{0}$; we have confirmed that the results presented below are essentially unchanged for $\left|\omega_{0}-2 \Omega_{0}\right|<<1$.

For the shear perturbations we are considering, $\boldsymbol{k} \cdot \delta \boldsymbol{v}_{n}=0$ and $\boldsymbol{k} \cdot \boldsymbol{v}_{p}=0$, that is, the velocity perturbations in the directions $\hat{y}$ and $\hat{e} \equiv-\cos \theta \hat{x}+\sin \theta \hat{z}$ are orthogonal to $\hat{k}$. We Fourier transform $\left(\propto \mathrm{e}^{i \mathbf{k} \cdot \mathbf{r}-i \sigma t}\right)$ eqs. 23]-28] and project the acceleration equations (23) and (25) onto $\hat{y}$ and $\hat{e}$. Defining $\sigma^{\prime} \equiv \sigma-k w_{0} \sin \theta, c \equiv \cos \theta, s \equiv \sin \theta, v_{B}^{2} \equiv B_{0} B_{c} / 4 \pi \rho_{p}$, $c_{B} \equiv \cos \left(\theta-\theta_{B}\right)$, and $x_{p} \equiv \rho_{p} / \rho_{n}$, we obtain the system of equations:

$$
\left[\begin{array}{cccc}
\mathcal{A}-i \sigma^{\prime}-i \epsilon_{n} k w_{0} s & -2 c \Omega_{0} \alpha & -\mathcal{A}+i \epsilon_{n} k w_{0} s & -2 \Omega_{0} c \beta^{\prime} \\
c\left(2 \Omega_{0} \alpha-i k w_{0} s \beta\right) & \left(\epsilon_{n}-1\right) i \sigma^{\prime}+2 \Omega_{0} c^{2} \beta-i k w_{0} s \beta^{\prime} & \mathcal{C} & -\mathcal{A}+i \epsilon_{n} k w_{0} s+2 \Omega_{0} s^{2} \beta \\
-x_{p}^{-1} \mathcal{A} & -2 x_{p}^{-1} \Omega_{0} c \beta^{\prime} & \mathcal{B} & 2 \Omega_{0} c\left(x_{p}^{-1} \beta^{\prime}-1\right) \\
x_{p}^{-1} \mathcal{C} & x_{p}^{-1}\left(-\mathcal{A}+2 \Omega_{0} s^{2} \beta\right) & 2 \Omega_{0} c-x_{p}^{-1} \mathcal{C} & \mathcal{B}-2 x_{p}^{-1} \Omega_{0} s^{2} \beta
\end{array}\right]\left[\begin{array}{c}
\hat{y} \cdot \delta \boldsymbol{v}_{n} \\
\hat{e} \cdot \delta \boldsymbol{v}_{n} \\
\hat{y} \cdot \boldsymbol{v}_{p} \\
\hat{e} \cdot \boldsymbol{v}_{p}
\end{array}\right]=0
$$

where 
$\mathcal{A} \equiv \epsilon_{n} i \sigma+2 \Omega_{0} \beta-i k w_{0} s \beta^{\prime}$

$\mathcal{B} \equiv-i \sigma+i v_{B}^{2} c_{B}^{2} k^{2} / \sigma+\nu_{e} k^{2}+x_{p}^{-1} \mathcal{A}$,

$\mathcal{C} \equiv c\left(i k w_{0} s \beta+2 \Omega_{0} \beta^{\prime}\right)$.

(Recall that $\beta^{\prime}=1-\alpha$ ). The speed of hydromagnetic waves is

$v_{B}=2 \times 10^{6}\left(\frac{\rho_{14}}{4}\right)^{-1 / 2}\left(\frac{x_{p}}{0.05}\right)^{-1 / 2} B_{0,12}^{1 / 2} \mathrm{~cm} \mathrm{~s}^{-1}$

significantly greater than the speed $w_{0}$ of the background flow (see eq. 14).

The dispersion relation that follows from eq. (30) is an impressively lengthy sixth-order polynomial in $\sigma$ in which all coefficients from sixth order to zeroth order are non-zero; it is easiest to explore various limits by working with eq. (30) directly. We first confirm a previous result of van Hoven \& Levin (2008) for perfect pinning $(\alpha=\beta=0)$, no background flow $\left(w_{0}=0\right)$, and no entrainment $\left(\epsilon_{n}=0\right)$. In this case, the polynomial factors into the form

$\sigma^{2}\left(\sigma^{4}+C \sigma^{3}+\left(-A^{2}+2 B+A C\right) \sigma^{2}+B C \sigma+B^{2}\right)=0$.

If we define

$f(\sigma)=\sigma^{2}+A \sigma+B=0$

and form the combination

$\sigma^{2}\left(f(\sigma)^{2}+(C-2 A) f(\sigma) \sigma\right)=0$

we obtain eq. (35), so two of the non-zero modes are given by the the simpler quadratic expression. Reading $A$ and $B$ from eq. (30) gives the dispersion relation

$\sigma^{2}+\left[-2 \Omega_{0}\left(1-\frac{1}{x_{p}}\right)+i \nu_{e} k^{2}\right] \sigma-\frac{4 \Omega_{0}^{2}}{x_{p}}-v_{B}^{2} k^{2}=0$,

as found by van Hoven \& Levin (2008) 1 For $\Omega_{0}=0$, the system has only damped hydromagnetic waves that travel at speed $v_{B}$.

We will not present here an analysis of the full mode structure of the system, but focus on two low-frequency modes that appear for imperfect pinning. For small $\alpha, \beta$, and $\epsilon_{n}$, the two zero-frequency modes of eq. (35) become small, and we can obtain these modes by working to second order in $\sigma$. Since $w_{0}$ is much smaller than $v_{B}$ (eqs. 14] and 34), we can further simplify the problem by taking the limit $v_{B} \rightarrow \infty$ for finite $w_{0}$. We take this limit by keeping only terms that multiply $v_{B}^{4}$, the highest order at which $v_{B}$ appears. With these approximations, eq. (30) gives

$$
\begin{aligned}
\left(1-\epsilon_{n}\right)^{2} \sigma^{2}+ & \left(2 \epsilon_{n}^{2} k w_{0} s+\epsilon_{n}\left\{-2 i \beta\left(1+c^{2}\right)-2 \beta^{\prime} k w_{0} s\right\}-2 \alpha k w_{0} s+2 i \beta\left(1+c^{2}\right)\right) \sigma \\
& -\left(\epsilon_{n} k w_{0} s\right)^{2}+\epsilon_{n}\left(2 \beta^{\prime}\left(k w_{0} s\right)^{2}+2 i \beta \Omega_{0} k w_{0} s\left(1+c^{2}\right)\right)-4\left(\alpha^{2}+\beta^{2}\right) \Omega_{0}^{2} c^{2}+\left(\alpha k w_{0} s\right)^{2}-2 i \alpha \beta k w_{0} s=0 .
\end{aligned}
$$

The electron viscosity does not appear at this level of approximation; in the $v_{B} \rightarrow \infty$ limit, the flux tube array is infinitely rigid, and vortex motion proceeds without producing shear in the proton-electron fluid. The validity of this approximation is confirmed below. The solutions to eq. (39) are

$\sigma_{ \pm}=\frac{1}{1-\epsilon_{n}}\left(\left(\alpha-\epsilon_{n}\right) k w_{0} \sin \theta-i \Omega_{0}\left(1+\cos ^{2} \theta\right) \beta \pm\left(4 \Omega_{0}^{2} \alpha^{2} \cos ^{2} \theta-\Omega_{0}^{2} \beta^{2} \sin ^{4} \theta-2 i \alpha \beta \Omega_{0} k w_{0} \cos ^{2} \theta \sin \theta\right)^{1 / 2}\right)$.

For $\theta=0$, the modes are

$\sigma_{ \pm}=\frac{2 \Omega_{0}}{1-\epsilon_{n}}( \pm \alpha-i \beta)$

Imperfect pinning has introduced two low-frequency modes to the system that are associated with slow vortex motion under the Magnus force. The modes are underdamped for $\beta<\alpha$, which defines the regime of low-drag creep that we will explore further.

The general solution with eigenvalue $\sigma_{-}$in eq. (40) is unstable above a critical wavenumber $k_{c}$. To find this wavenumber, we write the solution as

$\sigma_{-}=A+i B-\sqrt{C+i D}$

where

1 Our definition of $\sigma$ differs from that of van Hoven \& Levin (2008) by a minus sign. 
$A=\frac{\left(\alpha-\epsilon_{n}\right)}{1-\epsilon_{n}} k w_{0} s \quad B=-\frac{\Omega_{0}\left(1+c^{2}\right)}{1-\epsilon_{n}} \beta \quad C=\frac{4 \Omega_{0}^{2}}{\left(1-\epsilon_{n}\right)^{2}}\left(\alpha^{2} c^{2}-\beta^{2} s^{4}\right) \quad D=-\frac{2 \alpha \beta \Omega_{0}}{\left(1-\epsilon_{n}\right)^{2}} k w_{0} c^{2} s$

The solution becomes unstable when

$\operatorname{Im}\left(\sigma_{-}\right)=B-\operatorname{Im}(\sqrt{C+i D})=0$,

where

$\operatorname{Im}(\sqrt{C+i D})=(C+D)^{1 / 4} \sin \left(\frac{1}{2} \cos ^{-1}\left(\frac{C}{\sqrt{C^{2}+D^{2}}}\right)\right)$.

Combining this equation with eq. (44) gives

$4 B^{2}\left(B^{2}+C\right)=D^{2}$.

Solving for $k$ gives the critical wavenumber $k_{c}$ above which the system is unstable:

$k>k_{c} \equiv 2 \frac{\Omega_{0}}{w_{0}} \frac{\left(\beta^{2}+\alpha^{2}\right)^{1 / 2}}{\alpha} \frac{1+\cos ^{2} \theta}{\sin \theta \cos \theta}$,

independent of entrainment. The critical wavenumber $k_{c}$ is minimized for $\theta=\tan ^{-1}(\sqrt{2})$. For $k>>k_{c}$, we have the approximate solutions

$\sigma_{ \pm} \simeq \frac{1}{1-\epsilon_{n}}\left(\left(\alpha-\epsilon_{n}\right) k w_{0} \sin \theta \mp i\left(\alpha \beta \Omega_{0} k w_{0} \cos ^{2} \theta \sin \theta\right)^{1 / 2}\right) \quad k>>k_{c}$.

For the outer core, $\epsilon_{n} \simeq 3 \times 10^{-3}$ for $x_{p}=0.05$ (Chamel \& Haensel 2006). Though entrainment is essential in producing vortex pinning, it has a negligible effect on the growth rate of the instability, and no effect on the critical wavenumber, so we ignore it in the estimates below.

The instability arises from coupling between the velocity difference $\boldsymbol{w}_{n p}$ and the neutron vorticity $\boldsymbol{\omega}_{n}$ through the force of eq. 26. Dissipation damps perturbations for $k<k_{c}$, but for $k>k_{c}$ the finite vortex mobility gives rise to growing perturbations under the Magnus force. For $k>>k_{c}$, the growth rate scales as $\left(\alpha \beta w_{0}\right)^{1 / 2}$. For $\beta<<\alpha, k_{c}$ takes a constant value, but the growth rate of the mode becomes small, going to zero as $\beta$ goes to zero. In the highly-damped regime, $\beta>>\alpha$, damping restricts the unstable mode to large $k$, generally stabilizing the system. There are no unstable modes for either $\alpha=0$ or $\beta=0$; the instability occurs only if vortex motion has components along both $\hat{w}_{n p}$ and $\left(\hat{\omega}_{n} \times \hat{w}_{n p}\right) \times \hat{\omega}_{n}$.

We now confirm that the viscous stress is negligible for the $\sigma_{-}$unstable mode. The magnetic stress force per unit volume on the charged fluid from eq.(25) is

$\delta \boldsymbol{f}_{m} / \rho_{p}=\frac{\boldsymbol{B}_{\text {eff }, 0}}{4 \pi \rho_{p}} \cdot \nabla \delta \boldsymbol{B}_{\text {eff }}$,

while the viscous force is

$\boldsymbol{f}_{v} / \rho_{p}=\nu_{e} \nabla^{2} \boldsymbol{v}_{p}$

Fourier transforming, and using the induction equation (28), gives

$\frac{\delta f_{v}}{\delta f_{m}} \sim \frac{\nu_{e}}{v_{B}^{2}}\left(\frac{B_{c}}{B_{0}}\right)^{1 / 2} \operatorname{Re}\left(i \sigma_{-}\right)$.

This ratio is largest for high wavenumber. In this limit

$\frac{\delta f_{v}}{\delta f_{m}} \sim \Omega_{0} \frac{\nu_{e}}{v_{B}^{2}}\left(\frac{B_{c}}{B_{0}}\right)^{1 / 2}\left(\frac{w_{0}}{c_{T}}\right)^{1 / 2}(\alpha \beta)^{1 / 2}\left(\frac{k c_{T}}{\Omega_{0}}\right)^{1 / 2}$

In the outer core, $\nu_{e}$ is typically $\sim 10^{6} \mathrm{~cm}^{2} \mathrm{~s}^{-1}$ (Cutler \& Lindblom 1987; Andersson et al. 2005). Below we estimate $\alpha \beta \sim$ $10^{-18}$. The hydrodynamic treatment requires $k c_{T}<<\Omega_{0}$, so $\delta f_{v} / \delta f_{m} \lesssim 10^{-9}$ for a typical neutron star with $\Omega_{0}=100 \mathrm{rad} \mathrm{s}^{-1}$ and $B_{0}=10^{12} \mathrm{G}$; the viscous force is negligible compared to the magnetic force for these low-frequency modes.

\section{ESTIMATES OF THE INSTABILITY GROWTH RATE}

To obtain the growth rate of the instability, we now estimate the pinning parameters $\alpha$ and $\beta$ for the vortex creep process. To make these estimates, we regard the process of vortex creep as consisting of two distinct states of motion for a given vortex segment. Most of the time, the vortex segment is pinned. A small fraction of the time, the vortex segment is translating against a drag force to a new pinning configuration. The mutual friction force we are using (eq. 21) is

$\boldsymbol{f} / \rho_{n}=\boldsymbol{\omega}_{n} \times \boldsymbol{w}_{n p}-\alpha \boldsymbol{\omega}_{n} \times \boldsymbol{w}_{n p}+\beta \hat{\omega}_{n} \times\left(\boldsymbol{\omega}_{n} \times \boldsymbol{w}_{n p}\right)$. 
This force represents the spatially and temporally averaged force exerted on the neutron fluid by the creep process. The first term is the Magnus force for perfect pinning, while the remaining terms give the contribution to the force due to vortex motion. When a vortex segment is unpinned and moving against drag, we take the force to have the same form, but with different coefficients:

$\boldsymbol{f}_{0} / \rho_{n}=\boldsymbol{\omega}_{n} \times \boldsymbol{w}_{n p}-\alpha_{0} \boldsymbol{\omega}_{n} \times \boldsymbol{w}_{n p}+\beta_{0} \hat{\omega}_{n} \times\left(\boldsymbol{\omega}_{n} \times \boldsymbol{w}_{n p}\right)$.

An unpinned vortex segment remains unpinned for a time $t_{0} \sim d / w_{0}$, where $d$ is the distance the segment moves before repinning. This distance is comparable to the distance between pinning sites (Link et al. 1993). For pinning to flux tubes, $d \sim l_{\Phi} \simeq \sqrt{\Phi_{0} / B}$, and the average time that a vortex segment is unpinned is

$t_{0} \sim 10^{-14} B_{12}^{-1 / 2}\left(\frac{w_{0}}{10^{6} \mathrm{~cm} \mathrm{~s}^{-1}}\right)^{-1} \mathrm{~s}$,

much shorter than the hydrodynamic timescales of interest. Suppose that at any instant, the volume-averaged fraction of vortex length that is unpinned is $f_{v}<<1$. We now average $\boldsymbol{f}_{0}$ over a volume that contains many vortices, and over a time long compared to $t_{0}$ but short compared to hydrodynamic timescales, to obtain

$\left\langle\boldsymbol{f}_{0} / \rho_{n}\right\rangle=\boldsymbol{\omega}_{n} \times \boldsymbol{w}_{n p}-f_{v} \alpha_{0} \boldsymbol{\omega}_{n} \times \boldsymbol{w}_{n p}+f_{v} \beta_{0} \hat{\omega}_{n} \times\left(\boldsymbol{\omega}_{n} \times \boldsymbol{w}_{n p}\right)$.

Quantities related to the flow are unchanged by the averaging procedure since the superfluid flow velocity, the vorticity, and the relative flow velocity are independent of whether vortices are pinned or not. The factors of $f_{v}$ in eq. (56) account for the fact that only the motion of the translating vortex segments contributes to the mutual friction (see, also, Jahan-Miri 2006). For vortex motion by thermal activation, $f_{v} \sim \mathrm{e}^{-A / T}$, where $A$ is the activation energy for unpinning and $T$ is the temperature. The value of $f_{v}$ is unimportant for the following estimates.

The force of eq. (53), which is appropriate for vortex creep, must equal the average force $\left\langle\boldsymbol{f}_{0} / \rho_{n}\right\rangle$, giving the following relationships:

$\alpha=f_{v} \alpha_{0} \quad$ and $\quad \beta=f_{v} \beta_{0} \Rightarrow \frac{\beta}{\alpha}=\frac{\beta_{0}}{\alpha_{0}}$

We now take estimates of $\beta_{0} / \alpha_{0}$ to obtain the ratio $\beta / \alpha$.

The dominant drag process on unpinned vortex segments identified so far is the scattering of electrons against the magnetized cores of neutron vortices (Alpar et al. 1984b). For unpinned vortex segments, vortex motion can be described in terms of a single drag coefficient, as in eq. (22), so that

$1-\alpha_{0}=\frac{\mathcal{R}^{2}}{1+\mathcal{R}^{2}}=\mathcal{R} \beta_{0}$,

Using recent values of the proton effective mass, Siderv \& Alpar (2009) estimate

$\mathcal{R} \simeq 4 \times 10^{-4}-2 \times 10^{-5}$

giving $\alpha_{0} \simeq 1$ and $\beta_{0} \simeq \mathcal{R}$, so that

$\frac{\beta_{0}}{\alpha_{0}}=\frac{\beta}{\alpha} \simeq \mathcal{R}$

Vortex creep in the outer core is thus well into the low-drag regime. We stress that, in estimating $\beta_{0} / \alpha_{0}$, we have assumed that the drag relationship given by eq. (22) holds only for the small fraction $f_{v}$ of unpinned vortex length; the volume-averaged quantities $\beta$ and $\alpha$ satisfy $\beta / \alpha=\beta_{0} / \alpha_{0}$, from eq. (57), but there is no value of $\mathcal{R}$ that gives both $\beta<<\alpha$ and $\alpha<<1$, the creep regime of low dissipation in which we are interested.

We now estimate $\beta$. We adopt polar coordinates $(r, \phi, z)$, with the unperturbed vorticity along $\hat{z}$ and the unperturbed flow $\boldsymbol{w}_{0}$ along $\hat{\phi}$, and take the unperturbed flow and vortex velocity field to be axisymmetric. In the rotating frame $\left(\boldsymbol{v}_{p}=0\right)$, the unperturbed vortex velocity from eq. (20) is

$\boldsymbol{v}_{v, 0}=\alpha w_{0} \hat{\phi}+\beta w_{0} \hat{r}=\hat{n} v_{v, 0}$

where $\hat{n}$ is the average direction of vortex motion.

For steady spin down of the star, the core superfluid, the charged components, and the crust are spinning down at the same rate for a local differential velocity $w_{0}$. The creep velocity in this steady state is related to the spin-down rate by (Alpar et al. 1984a; Link et al. 1993)

$\dot{\Omega}=-2 \frac{\Omega}{r} \boldsymbol{v}_{v, 0} \cdot \hat{r}=-2 \frac{\Omega}{r} w_{0} \beta=\dot{\Omega}_{0}$,

where $\Omega$ is the spin rate of the superfluid, $\dot{\Omega}_{0}$ is the observed spin down rate of the crust, and $r$ is approximately the stellar radius $R$. We arrive at the estimate 
$\beta=\frac{R}{4 w_{0} t_{\mathrm{age}}} \simeq 10^{-11}\left(\frac{w_{0}}{10^{5} \mathrm{~cm} \mathrm{~s}^{-1}}\right)^{-1}\left(\frac{t_{\mathrm{age}}}{10^{4} \mathrm{yr}}\right)^{-1}$.

where $\Omega \simeq \Omega_{0}$ is assumed. Eq. (63), in combination with eq. (57), gives the fiducial values $\alpha=10^{-7}$ and $\alpha \beta=10^{-18}$. For these values, we deduce $f_{v} \sim\left(\alpha \beta / \alpha_{0} \beta_{0}\right)^{1 / 2} \sim 10^{-6}$, that is, most of the vortex length is pinned at any instant.

We can now proceed with estimates of the instability length scale and growth rate. For $\alpha>>\beta$ and $\theta=\tan ^{-1}(\sqrt{2})$ in eq. (47), the critical wavenumber is

$k_{c} \simeq 6 \frac{\Omega}{w_{0}}=6 \times 10^{-3}\left(\frac{\Omega}{100 \mathrm{rad} \mathrm{s}^{-1}}\right)\left(\frac{w_{0}}{10^{5} \mathrm{~cm} \mathrm{~s}^{-1}}\right)^{-1}$,

corresponding to a wavelength $\lambda=2 \pi / k \simeq 10 \mathrm{~m}$. For $k>>k_{c}$, the growth rate from eq. (48) is

$\frac{1}{2 \pi} \operatorname{Im}\left(\sigma_{-}\right) \simeq 2\left(\frac{\alpha \beta}{10^{-18}}\right)^{1 / 2}\left(\frac{\Omega}{100 \mathrm{rad} \mathrm{s}^{-1}}\right)^{1 / 2}\left(\frac{w_{0}}{10^{5} \mathrm{~cm} \mathrm{~s}^{-1}}\right)^{1 / 2}\left(\frac{\lambda}{1 \mathrm{~m}}\right)^{-1 / 2} \mathrm{yr}^{-1}$

The hydrodynamic treatment is restricted to $c_{T} k<<\Omega$. To estimate how high the growth rate could be, we consider a maximum wavenumber defined by $c_{T} k_{\max }=0.1 \Omega$, where $c_{T} \simeq 10^{-1}\left(\Omega / 100 \mathrm{rad} \mathrm{s}^{-1}\right)^{1 / 2} \mathrm{~cm} \mathrm{~s}^{-1}$. The growth rate at this wavenumber, from eq. (48), is

$\frac{1}{2 \pi} \operatorname{Im}\left[\sigma_{-}\left(k_{\max }\right)\right] \simeq 0.2\left(\frac{\alpha \beta}{10^{-18}}\right)^{1 / 2}\left(\frac{\Omega}{100 \mathrm{rad} \mathrm{s}^{-1}}\right)^{3 / 4}\left(\frac{w_{0}}{10^{5} \mathrm{~cm} \mathrm{~s}^{-1}}\right)^{1 / 2} \mathrm{~d}^{-1}$

For $\Omega=100 \mathrm{rad} \mathrm{s}^{-1}$, the corresponding wavenumber is $k \simeq 100 \mathrm{~cm}^{-1}$. Eq. (66) does not represent a physical limit, but only the restrictions of the hydrodynamic treatment; the instability could continue to exist also for wavenumbers in the regime $k c_{T}>\Omega$. If vortex creep is in the strongly-damped regime $\beta>>\alpha$, contrary to the estimates here, there is still a broad window for instability. Requiring $k_{c}<k_{\max }$ gives

$\beta<2 \times 10^{4}\left(\frac{w_{0}}{10^{5} \mathrm{~cm} \mathrm{~s}^{-1}}\right)\left(\frac{\Omega}{100 \mathrm{rad} \mathrm{s}^{-1}}\right)^{-1 / 2} \alpha$

and the star will be unstable at some wavenumber that is consistent with the hydrodynamic approximation $c_{T} k<<\Omega$.

\section{TIMING NOISE DRIVEN BY SUPERFLUID TURBULENCE?}

The superfluid flow could quickly become turbulent through the instability identified here. Turbulent flow would exert random torques on the crust, possible contributing to observed timing anomalies such as timing noise and glitches. We now estimate the spectrum of turbulent torque fluctuations using the similarity argument of Kolmogorov (1941) and Obukhov (1941). In this argument, the properties of the turbulent spectrum follow from the assumption that turbulent energy is being dissipated at a rate $\dot{\epsilon}$ per unit mass at some high wavenumber.

The torque fluctuation per unit mass of fluid for a mode of wavenumber $k$ is

$\delta N(k) d k \simeq \pm l(\Delta v) f$

where $l \sim 1 / k$ is the characteristic dimension of a turbulent cell, and $\Delta v$ is the characteristic change in flow speed over a time $1 / f$, and $f l \sim \Delta v$. The units of $\delta N(k)$ are $\mathrm{cm}^{3} \mathrm{~s}^{-2}$, and the only possible combination of $\dot{\epsilon}$ and $k$ with the correct units is

$\delta N(k)= \pm A(\dot{\epsilon})^{2 / 3} k^{-5 / 3}$,

where $A$ is a dimensionless constant. The time average of $\delta N(k)$ is zero.

Integrated over the star of radius $R$, the contributions to the total torque by different cells of wavenumber $k$ add incoherently, and the fluctuation in the total torque at wavenumber $k$ scales as

$\delta N_{\text {tot }}(k) \propto \sqrt{M} \delta N(k)$,

where $M$ is the characteristic number of turbulent cells of wavenumber $k$,

$M=\left(\frac{R}{l}\right)^{d}$.

Here $d$ is the dimensionality of the turbulence. Isotropic turbulence corresponds to $d=3$, while highly-polarized turbulence corresponds to $d=1$. The turbulence should be polarized to some extent by rotation (see, e.g., Andersson et al. 2007).

The fluctuations in the total torque scale as

$\delta N_{\text {tot }}(k) \propto \pm k^{d / 2} k^{-5 / 3}$,

and the spectral power of torque fluctuations (spectral index $\alpha_{t}$ ) is 


\begin{tabular}{lll}
\hline SOURCE & SPECTRAL INDEX & REFERENCE \\
\hline PSR 0823+26 & $\alpha_{t}=-1.16 \pm 0.48$ & Bavkal et al. (1999) \\
& $\alpha_{t}=-0.39 \pm 0.36$ & \\
PSR 2021+51 & $\alpha_{t}=-1.95 \pm 0.74$ & Bavkal et al. (1999) \\
& $\alpha_{t}=-1.64 \pm 1.40$ & \\
PSR 1706-16 & $\alpha_{t}=-2.41 \pm 0.79$ & Bavkal et al. (1999) \\
& $\alpha_{t}=-1.94 \pm 1.33$ & \\
PSR 1749-28 & $\alpha_{t}=-0.88 \pm 0.05$ & Bavkal et al. (1999) \\
& $\alpha_{t}=-1.05 \pm 0.83$ & \\
PSR B1509-58 & $\alpha_{p}=-4.6 \pm 1.0$ & Livingstone et al. (2005) \\
PSR B0628-28 & $\alpha_{p} \simeq-1(t<2 \mathrm{yr})$ & Hobbs et al. (2010) \\
PSR B0628-28 & $\alpha_{p} \simeq-5(t>2 \mathrm{yr})$ & Hobbs et al. (2010) \\
SGR 1900+14 & $\alpha_{t}=-3.7 \pm 0.6$ & Woods et al. (2002) \\
SGR 1806-20 & $\alpha_{t}=-3.6 \pm 0.7$ & Woods et al. (2002) \\
\hline
\end{tabular}

Table 1. Estimates of spectral indices. The second entry for PSRs $0823+26,2021+51,1706-16$, and $1759-29$ follows from a fit to the arrival times to a higher-order polynomial; see Baykal et al. 1999 for details. PSR B0628-28 shows $\alpha_{p} \simeq-1$ over timescales of less than two years, and $\alpha_{p} \simeq-5$ over longer timescales.

$\frac{d\left|\delta N_{\text {tot }}(f)\right|^{2}}{d f} \propto f^{-13 / 3+d} \equiv f^{\alpha_{t}} \longrightarrow f^{-3.3}$ to $f^{-1.3}$

Let the rotation phase of a pulsar be $\phi(t)$, so that $d \phi(t) / d t$ is the spin rate. A torque variation $\delta N_{\text {tot }}(t)$ produces a variation in the rotational phase $\delta \phi(t)$, where

$\delta N_{\text {tot }}(t)=2 \pi I_{c} \frac{d^{2} \delta \phi}{d t^{2}}$

and $I_{c}$ is the moment of inertia of the crust plus any other components of the star tightly coupled to the crust. The spectrum of phase residuals $\delta \phi$ (spectral index $\alpha_{p}$ ) is therefore

$\frac{d|\delta \phi(f)|^{2}}{d f} \propto f^{-25 / 3+d} \equiv f^{\alpha_{p}} \longrightarrow f^{-7.3}$ to $f^{-5.3}$

"Torque noise" corresponds to $\alpha_{p}=-5$. We conclude that turbulence can produce such a power spectrum only if the turbulence is nearly isotropic $(d=3)$. From the scaling argument given here, turbulence cannot produce "frequency noise" $\left(\alpha_{p}=-3\right)$ nor "phase noise" $\left(\alpha_{p}=-1\right)$. In early work on 45 pulsars, D'Alessandro et al. (1995) reported power spectra consistent with a range $-1 \leq \alpha_{p}<-5$. More recent estimates are given in Table 1 for six radio pulsars and two magnetars. These results should be regarded as illustrative only; the power spectra of many pulsars do not follow simple power laws (Cordes \& Downs 1985), and some sources show quasi-oscillatory behavior; see Hobbs et al. (2010) for a discussion of these issues. Though timing noise is a complicated process that is difficult to characterize, we note that the basic power spectra predicted by turbulent torque fluctuations are generally consistent with observed power spectra.

\section{DISCUSSION AND CONCLUSIONS}

We have identified a dissipation-driven instability that could operate in the fluid of the neutron star core over length scales shorter than $\sim 10 \mathrm{~m}$ and over timescales as fast as days. The instability is a result of the forcing the vortices against the rigid array of flux tubes. The superfluid flow of the in the outer core could become turbulent if dissipative processes do not suppress the instability. The excitation of Kelvin waves vortices (Link 2004) will lead to dissipation, but is probably not nearly strong enough to put vortex creep into the highly-dissipative regime that would be required to quench the instability; this process damps rotational perturbations over many spin periods, and so is in the low-drag regime. Other dissipation mechanisms of possible relevance require study. If the core fluid becomes turbulent, the closest experimental analogue might be grid turbulence that has been well studied in superfluid helium (Smith et al. 1993). In our previous work on the crust Link 2012), we found that the flow is unstable there as well. We conclude that superfluid flow throughout much of a neutron star is forced unstable by the pinning stresses on the fluid that arise as the star spins down.

To illustrate the basic instability, we have taken a simplified mutual friction force in which the pinning coefficients $\alpha$ and $\beta$ are constants. The estimates of the instability growth rate given here should be regarded as rough estimates. For thermally-activated vortex creep, the pinning coefficients will have exponential dependence on the scalar $w_{n p}$ (Alpar et al. 1984a; Link et al. 1993). We expect that this strong velocity dependence will significantly enhance the growth rate of the instability, without significantly changing the critical wavenumber so long as vortex motion is in the regime of low drag. Further work is needed on this issue. 
Turbulent flow could create torque fluctuations with spectra that are consistent with those measured in many radio pulsars and in magnetars. While the simple scaling argument given in $\S 5$ suggests that turbulent torques cannot produce "phase noise" or "frequency noise", it would be interesting to further explore the possibility that steeper power spectra result from turbulent torques. In future work we will determine if the instability identified in this paper leads to fully-developed turbulence, and estimate the magnitude of the fluctuations.

\section{ACKNOWLEDGMENTS}

We thank the anonymous referee for particularly useful constructive criticism.

\section{REFERENCES}

Alpar M. A., Anderson P. W., Pines D., Shaham J., 1984a, Astrophys. J., 276, 325

Alpar M. A., Langer S. A., Sauls J. A., 1984b, Astrophys. J., 282, 533

Andersson N., Comer G. L., 2006, Classical and Quantum Gravity, 23, 5505

Andersson N., Comer G. L., Glampedakis K., 2005, Nucl. Phys. A, 763, 212

Andersson N., Comer G. L., Prix R., 2004, Mon. Not. Roy. Astron. Soc., 354, 101

Andersson N., Sidery T., Comer G. L., 2007, Mon. Not. Roy. Astron. Soc., 381, 747

Baykal A., Alpar M. A., Boynton P. E., Deeter J. E., 1999, Mon. Not. Roy. Astron. Soc., 306, 207

Baym G., Pethick C., Pines D., 1969, Nature, 224, 673

Chamel N., Haensel P., 2006, Phys. Rev. C, 73, 045802

Chau H. F., Cheng K. S., Ding K. Y., 1992, Astrophys. J., 399, 213

Cordes J. M., Downs G. S., 1985, Astrophys. J. Suppl., 59, 343

Cutler C., Lindblom L., 1987, Astrophys. J., 314, 234

D'Alessandro F., McCulloch P. M., Hamilton P. A., Deshpande A. A., 1995, Mon. Not. Roy. Astron. Soc., 277,1033

Elgarøy Ø., Engvik L., Hjorth-Jensen M., Osnes E., 1996, Phys. Rev. Lett., 77, 1428

Glaberson W. I., Johnson W. W., Ostermeier R. M., 1974, Phys. Rev. Lett., 33, 1197

Glampedakis K., Andersson N., 2009, Phys. Rev. Lett., 102, 141101

Glampedakis K., Andersson N., Jones D. I., 2008a, Mon. Not. Roy. Astron. Soc., 394, 1908

Glampedakis K., Andersson N., Jones D. I., 2008b, Phys. Rev. Lett., 100, 081101

Glampedakis K., Andersson N., Samuelsson L., 2011, Mon. Not. Roy. Astron. Soc., 410, 805

Hall H. E., Vinen W. F., 1956, Proc. R. Soc. A, 238, 215

Haskell B., 2011, Phys. Rev. D, 83, 043006

Hobbs G., Lyne A. G., Kramer M., 2010, Mon. Not. Roy. Astron. Soc., 402, 1027

Jahan-Miri M., 2006, Astrophys. J., 650, 326

Jones P. B., 1991, Mon. Not. Roy. Astron. Soc., 253, 279

Kolmogorov A. N., 1941, Dokl. Acad. Nauk. SSSR, 30, 301

Link B., 2004, Phys. Rev. Lett., 92, 149002

Link B., 2009, Phys. Rev. Lett., 102, 131101

Link B., 2012, Mon. Not. Roy. Astron. Soc. in press (arXiv:1105.4654)

Link B., Epstein R. I., Baym G., 1993, Astrophys. J., 403, 285

Livingstone M. A., Kaspi V. M., Gavriil F. P., Manchester R. N., 2005, Astrophys. J., 619, 1046

Melatos A., Peralta C., 2007, Astrophys. J., 662, L99

Mendell G., 1991, Astrophys. J., 380, 515

Migdal A. B., 1959, Nucl. Phys., 13, 655

Noronha J., Sedrakian A., 2008, Phys. Rev. D, 77, 023008

Obukhov A. M., 1941, Dokl. Acad. Nauk. SSSR, 32, 22

Peralta C., Melatos A., 2009, Astrophys. J., 701, L75

Peralta C., Melatos A., Giacobello M., Ooi A., 2005, Astrophys. J., 635, 1224

Peralta C., Melatos A., Giacobello M., Ooi A., 2006, Astrophys. J., 651, 1079

Prix R., 2004, Phys. Rev. D, 69, 043001

Ravenhall D. G., Pethick C. J., Wilson J. R., 1983, Phys. Rev. Lett., 50, 2066

Ruderman M., Zhu T., Chen K., 1998, Astrophys. J., 492, 267

Schulze H.-J., Cugnon J., Lejeune A., Baldo M., Lombardo U., 1996, Phys. Rev. Lett., 375, 1

Sidery T., Alpar M. A., 2009, Mon. Not. Roy. Astron. Soc., 400, 1859

Sidery T., Andersson N., Comer G. L., 2008, Mon. Not. Roy. Astron. Soc., 385, 335 
Sjöberg O., 1976, Nucl. Phys. A, 65, 511

Smith M. R., Donnelly R. J., Goldenfeld N., Vinen W. F., 1993, Phys. Rev. Lett., 71, 2583

Tkachenko V. K., 1966a, Zh. Eksp. Teor. Fiz., 50, 1573

Tkachenko V. K., 1966b, Sov. Phys. JETP, 23, 1049

van Hoven M., Levin Y., 2008, Mon. Not. Roy. Astron. Soc., 391, 283

Woods P. M., Kouveliotou C., Göğüs E., Finger M., Swank J., Markwardt C. B., Hurley K., van der Klis M., 2002, Astrophys. J., 576,381 